Pathologe 2008 · [Suppl 2] 29:353-356 DOI 10.1007/s00292-008-1056-5

Online publiziert: 20. August 2008

๑) Springer Medizin Verlag 2008

S. Aulmann · Z. Elsawaf · R. Penzel · P. Schirmacher $\cdot$ H.P. Sinn

Pathologisches Institut, Universitätsklinikum Heidelberg

\title{
Klonaler Zusammenhang flacher Epithelatypien und tubulärer Mammakarzinome
}

deutet mikropapilläre büschelartige Ausstülpungen in das Lumen der okkupierten Ganganschnitte aufweist. Die Unterscheidung von FEA gegenüber Läsionen ohne Atypien erfolgt im Wesentlichen anhand nukleärer Veränderungen. Dabei zeigen FEA mehrheitlich runde, gelegentlich aber auch ovale Kerne mit aufgehobener Kernpolarisation und kleinen $\mathrm{Nu}$ kleolen [16].

Während derartige subtile Veränderungen in der Umgebung invasiver Karzinome über lange Zeit wenig beachtet wurden, hat sich im Rahmen der immer besseren Bildgebung und der inzwi- schen weit verbreiteten Früherkennungsprogramme die Aufmerksamkeit der klinisch-pathologischen Forschung auch auf die frühen möglichen Vorläuferläsionen ausgedehnt. Auch wenn bereits Azzopardi [3] das gleichzeitige Auftreten tubulärer Mammakarzinome mit monomorphen "Clinging-Karzinomen" und Herden eines LCIS erwähnte [3], dauerte es bis 1998, bis die häufige Koinzidenz der Veränderungen in mehreren Veröffentlichungen dargestellt wurde [9]. Aufgrund der hohen zytologischen Ähnlichkeit zwischen FEA und tubulären Mammakarzinomen wurde von mehreren Autoren eine wie „blunt duct adenosis“ (BDA), prätubuläre Hyperplasie, Zylinderzellmetaplasie, „clinging carcinoma in situ“ oder auch duktale intraepitheliale Neoplasie, „flat type" [10]. Derzeit unterscheidet die am weitesten verbreitete Klassifikation nach O'Malley et al. [16] 3 wesentliche Typen zylinderepithelialer Veränderungen:

- Zylinderzelläsionen ohne Atypien,

- Zylinderzellhyperplasien ohne Atypien und

- flache Epithelatypien (FEA).

Histologisch sind erstere durch ein einoder zweireihiges Zylinderepithel mit uniformen, ovoiden oder elongierten, entlang der Basalmembran ausgerichteten Zellkernen gekennzeichnet. Apikale Zellfortsätze (,snouts") sind wie auch in anderen CCL (Zylinderzellläsionen) oft nachweisbar. Zylinderzellhyperplasien $(\mathrm{CCH})$ zeigen bei ähnlicher Zytologie ein mehr als zweireihiges Epithel, das oft auch ange-

\begin{tabular}{|c|c|c|c|c|}
\hline Nr. & Alter (Jahre) & TNM (2005) & $\begin{array}{l}\text { Klonalität } \\
\text { FEA/TUB }\end{array}$ & $\begin{array}{l}\text { Weitere } \\
\text { Läsionen }\end{array}$ \\
\hline 1 (4996) & 51 & pT1a, pNx, G1 & Nein & \\
\hline $2(6210)$ & 56 & pT1b, pN1biii, G1 & Nein & DCIS \\
\hline 3 (9039) & 52 & pT2, pN0, G1 & Nein & DCIS \\
\hline $4(10348)$ & 66 & pT1b, pNx, G1 & Ja & \\
\hline $5(13285)$ & 59 & pT1c, pN0, G1 & Nein & \\
\hline $6(16713)$ & 59 & pT1c, pN0, G1 & (Nicht informativ) & \\
\hline $7(17245)$ & 55 & pT1c, pN0, G1 & Ja & \\
\hline $8(21140)$ & 66 & pT1b, pN0, G1 & Ja & \\
\hline 9 (29904) & 72 & pT1c, pN0, G1 & Ja & \\
\hline $10(40227)$ & 54 & pT2, pN0, G1 & Nein & \\
\hline 11 (1048) & 57 & pT1c, pN0, G1 & Nein & DCIS \\
\hline $12(4607)$ & 48 & pT1c, pN0, G1 & $\mathrm{Ja}$ & DCIS \\
\hline $13(3739)$ & 60 & pT1b, pN0, G1 & Nein & LCIS \\
\hline $14(3871)$ & 62 & pT1c, pN0, G1 & $\mathrm{Ja}$ & \\
\hline $15(3583)$ & 63 & pT1c, pN0, G1 & $\mathrm{Ja}$ & \\
\hline $16(3173)$ & 62 & pT1b,pN0,G1 & Nein & LCIS \\
\hline
\end{tabular}




\section{Beste Forschungsbeiträge: Gynäkopathologie}

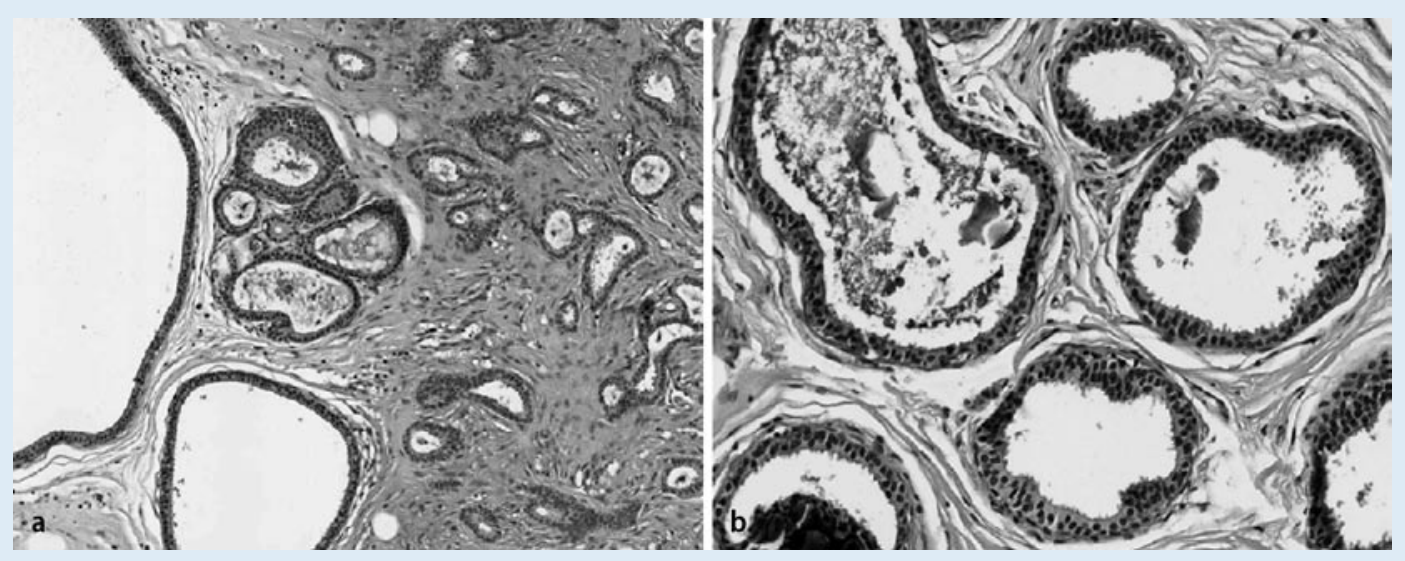

Abb. $1<$ a Tubuläres Mammakarzinom mit begleitenden FEA. b Höhere Vergrößerung von FEA: terminale Ganganschnitte, ausgekleidet von einer ein- bis zweireihigen Lage zylindrischer Epithelien mit prominenten Zellfortsätzen („snouts“), aufgehobener Kernpolarisation und geringgradigen Kernatypien. Damit assoziiert einzelne Mikroverkalkungen
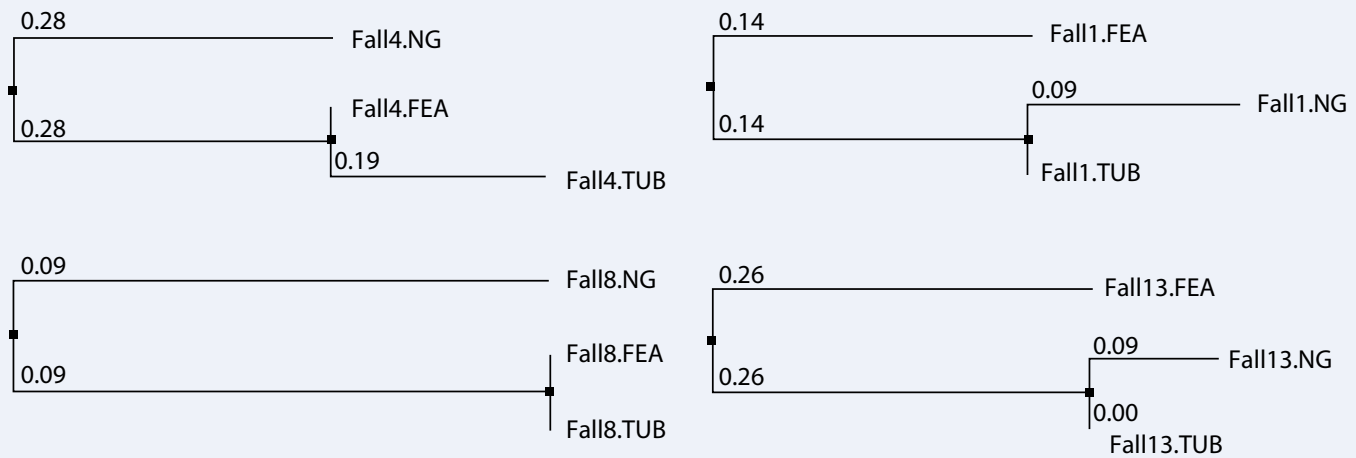

Abb. $2 \Delta$ Beispiele von Dendrogrammen der mtDNA-basierten Klonalitätsanalyse. Links Fälle mit klonalem Zusammenhang zwischen FEA und tubulären Karzinomen. Rechts 2 Beispiele ohne nachweisbaren Zusammenhang. Die mit Hilfe der „Neighbour-joining-Methode" errechneten relativen Distanzen sind als kleine Zahlen neben den Abzweigungen der Dendrogramme angegeben

eventuelle Vorläuferrolle diskutiert [7, 8]. Um diese näher zu untermauern, haben wir an einem kleinen Kollektiv tubulärer Karzinome und assoziierter FEA Klonalitätsanalysen auf der Basis mitochondrialer DNA-Sequenzierung durchgeführt.

\section{Methoden}

\section{Patientenkollektiv}

Eine initiale Serie von 20 konsekutiven tubulären Mammakarzinomen aus den Jahren 2000 und 2001, die in der Universitätsfrauenklinik Heidelberg operiert worden waren, wurde aus den Archiven des Pathologischen Institutes der Universität Heidelberg herausgesucht und auf das Vorhandensein von FEA hin nachmikroskopiert. Zusätzlich wurden weitere beglei- tende nichtinvasive Veränderungen sowie Tumorstadium, Patientenalter und der Rezeptorstatus aus der Datenbank der Sektion für gynäkologische Pathologie extrahiert.

\section{DNA-Isolation}

Jeweils 4 Paraffinschnitte $(8 \mu \mathrm{m})$ von 16 Fällen mit FEA und begleitenden tubulären Karzinomen sowie normalen Drüsenläppchen aus der unmittelbaren Umgebung wurden auf unbeschichtete Objektträger aufgezogen, entparaffiniert und rehydriert. Danach wurden jeweils einzelne Läsionen mit Hilfe von Glaskapillaren mikrodisseziert, in Reaktionsgefäße mit $20 \mu$ Isolationspuffer $(50 \mathrm{mM}$ Tris $/ \mathrm{HCl}$, 10 mM EDTA, 0,05\% Tween 20, $1 \%$ Proteinase $\mathrm{K}, \mathrm{pH}$ 8) überführt und bei $65^{\circ} \mathrm{C}$ über Nacht lysiert. Nach Hitzeinaktivierung der Proteinase $\mathrm{K}$ wurde der Überstand direkt für die nachfolgenden Polymerasekettenreaktionen (PCR) verwendet.

\section{Sequenzanalyse der mitochondrialen Kontrollregion}

DNA der mitochondrialen Kontroll- oder D-Loop-Region (Nukleotide 16045-650) wurde mittels PCR in 2 Fragmenten, wie von Morandi et al. [15] beschrieben, amplifiziert. Nach gelelektrophoretischer Kontrolle wurden Primer und nichtinkorporierte Nukleotide mit Hilfe eines säulenbasierten PCR-Aufreinigungssystems entfernt (High-Pure PCR Cleanup Kit, Roche, Mannheim). Im Anschluss erfolgte die bidirektionale direkte DNA-Se- 
quenzierung mittels des DYenamic ET Sequencing Kits (GEHealthcare, Freiburg) auf einem ABI Prism 377 Sequencer (Applied Biosystems/Applera, Darmstadt). Für die phylogenetische Analyse der mtDNA-Sequenzen wurde das webbasierte ClustalW-Softwarepaket verwendet [5]. Dendrogramme mit Angabe der relativen Distanzen wurden mit Jalview 2.3 [6] erstellt.

\section{Ergebnisse}

Von den 20 primär untersuchten tubulären Mammakarzinomen wiesen 16 Herde von FEA in unmittelbarer Tumornähe auf. In 4 dieser Präparate fanden sich daneben DCIS, 2 Fälle zeigten Herde eines LCIS. Von den übrigen 4 tubulären Karzinomen fanden sich in 2 begleitend hochdifferenzierte DCIS sowie einfache CCL ohne Atypien bzw. CCH ohne Atypien in jeweils einem Fall.

Das mediane Erkrankungsalter betrug 58 Jahre. Zwei Karzinome wiesen zum Zeitpunkt der Diagnose eine Größe von mehr als $2 \mathrm{~cm}$ auf, lediglich in einem Fall fanden sich axilläre Lymphknotenmetastasen. Sämtliche Karzinome zeigten eine kräftige Expression des Östrogen- und Progesteronrezeptors, eine Her2-Überexpression lag in keinem Tumor vor. Die klinisch-pathologischen Daten sind in - Tab. 1 zusammengefasst, $\bullet$ Abb. 1 a, b zeigt exemplarische Fälle.

Die Sequenzanalyse der hochvariablen Region der mitochondrialen DNA (mtDNA) zeigte im Vergleich zu der untersuchten DNA aus normalen Drüsenläppchen in 15 Fällen Sequenzabweichungen. Lediglich in Fall 6 erbrachte die Klonalitätsanalyse aufgrund vollständig übereinstimmender Sequenzen in tubulärem Karzinom, FEA und normalem Drüsengewebe kein informatives Ergebnis. Die beobachtete Anzahl an Mutationen betrug zwischen 1 und 17 (Median: 5). Im Falle divergierender Mutationen wurde die Untersuchung als nicht klonal gewertet, bei übereinstimmenden Mutationen (Fälle 4, 7, 8, 9, 12, 14 und 15) als klonal. In einem dieser Fälle (Fall 12) wies das tubuläre Karzinom neben 2 mit der FEA übereinstimmende noch 2 zusätzliche Mutationen auf. In Fall 4 bestanden neben 6 übereinstim- menden Mutationen im tubulären Karzinom 3 weitere Sequenzaberrationen.

Somit ließ sich von den 15 informativen Fällen in 7 Paaren aus FEA und tubulären Karzinomen ein klonaler Zusammenhang (47\%) nachweisen. Zusätzlich zeigten 2 weitere Fälle (Fall 10 und 11) eine hohe Sequenzhomologie mit 7 bzw. 9 übereinstimmenden Mutationen. Da jedoch die untersuchten FEA-Foci in Fall 10 drei und in Fall 11 fünf zusätzliche Mutationen im Vergleich zum tubulären Karzinom zeigten, kann in diesen Fällen kein eindeutiger klonaler Zusammenhang als gesichert gelten. - Abb. 2 zeigt Beispiele der aus der phylogenetischen ClusterAnalyse erzeugten Dendrogramme, die Auswertung der Klonalitätsanalyse ist in - Tab. 1 zusammengefasst.

\section{Diskussion}

Während CCL der Mamma bereits seit Jahrzehnten bekannt sind, haben sie dennoch erst in jüngster Vergangenheit Aufmerksamkeit als mögliche Vorläuferläsionen invasiver Karzinome erlangt. Insbesondere das gleichzeitige Auftreten von CCL mit tubulären Karzinomen und ihre große zytologische Ähnlichkeit wurde von mehreren Autoren als möglicher Hinweis auf einen biologischen Zusammenhang beschrieben $[9,11]$. Der Nachweis rekurrenter chromosomaler Imbalancen in CCL deutete auf ein klonales und neoplastisches Geschehen hin [19] und widersprach damit früheren Einschätzungen dieser Läsionen als einfache hyperplastische Veränderungen. Interessanterweise bestehen große Ähnlichkeiten in den häufig alterierten chromosomalen Regionen zwischen CCL, LCIS und (low grade) DCIS [12, 13, 14]. Auf der Basis der beobachteten chromosomalen Imbalancen und des morphologischen Spektrums der CCL, CIS und assoziierter invasiver Tumoren schlugen Abdel-Fatah et al. [1] ein sequenzielles Modell der Entstehung hochdifferenzierter Mammakarzinome vor.

Die Untersuchung von mtDNA-Sequenzen ermöglicht aufgrund der durch das Fehlen spezifischer DNA-Reparaturenzyme bedingen hohen Spontanmutationsrate insbesondere in der hochvariablen Kontroll- oder D-Loop-Region Ein-
Pathologe 2008 · [Suppl 2] 29:353-356

DOI 10.1007/s00292-008-1056-5

(C) Springer Medizin Verlag 2008

S. Aulmann · Z. Elsawaf · R. Penzel · P. Schirmacher $\cdot$ H.P. Sinn

Klonaler Zusammenhang flacher Epithelatypien und tubulärer Mammakarzinome

Zusammenfassung

Flache Epithelatypien (FEA) werden derzeit als mögliche Vorläufer hochdifferenzierter Mammakarzinome diskutiert. Insbesondere tubuläre Karzinome treten oft in unmittelbarer Beziehung mit FEA auf und zeigen auch zytologisch eine große Ähnlichkeit. Um einen möglichen klonalen Zusammenhang zu untersuchen, haben wir eine Mutationsanalyse der hochvariablen Region des mitochondrialen Genoms in einer Serie tubulärer Karzinome, FEA und benachbarter normaler Drüsenläppchen durchgeführt. Mittels „,multiple sequence alignment" ergaben sich identische mtDNA-Mutationen in 50\% der Fälle als Hinweis auf einen klonalen Zusammenhang. Diese Ergebnisse deuten auf eine mögliche Vorläuferrolle von FEA in der Entstehung tubulärer Mammakarzinome hin.

Schlüsselwörter

Flache Epithelatypien · Tubuläre Mammakarzinome · Mitochondriale DNA · Vorläuferläsionen · DNA-Sequenzierung

\section{Clonal association of flat epithelial atypia and tubular breast cancer}

Abstract

Flat epithelial atypia (FEA) of the breast has recently gained attention as a possible precursor lesion of highly differentiated breast cancer. Especially tubular carcinomas, with which FEA shares cytological features, often occur in close proximity to each other. To examine a possible clonal relationship, we analysed mutations of the highly variable region of the mitochondrial genome in a series of tubular carcinomas, associated FEA and normal glands. Multiple sequence alignment showed identical mtDNA mutations in approximately $50 \%$ of paired FEA and tumour samples, indicative of a clonal relationship. Our data indicate a possible precursor role of FEA in the development of tubular breast cancer.

Keywords

Flat epithelial atypia - Tubular carcinoma . Mitochondrial DNA · Precursor lesions · DNA sequencing 
blicke in die biologischen Zusammenhänge unterschiedlicher Läsionen [15]. In der Vergangenheit konnten wir bereits mit Hilfe dieser Methode eine Vorläuferrolle von LCIS für die Entstehung invasiver lobulärer Karzinome nachweisen [2]. Die hier vorgestellten Untersuchungen tubulärer Karzinome und assoziierter FEA konnten in etwa der Hälfte der Fälle einen klonalen Zusammenhang beider Läsionen nachweisen. Da CCL oder FEA häufig multifokal auftreten [19], erscheint es möglich, dass mit der Untersuchung weiterer Herde von FEA in den übrigen 8 Fällen die Nachweisrate noch zu steigern gewesen wäre. Der in einem Teil der Fälle nachgewiesene klonale Zusammenhang spricht jedoch schon jetzt für eine Vorläuferrolle von FEA. Zusätzlich ist die beobachtete häufige Assoziation von CCL mit oder ohne Atypien mit lobulären Neoplasien oder (low grade) DCIS sowie die hohe Rate multifokaler oder auch bilateraler Läsionen möglicherweise ein Ausdruck einer generell bestehenden erhöhten neoplastischen Transformationstendenz im Sinne einer Feldkanzerisierung.

\section{Fazit}

Zusammenfassend scheinen FEA, ähnlich wie lobuläre Neoplasien, ein doppeltes Spiel in der mammären Tumorenstehung zu spielen. Sowohl die beobachteten klinisch-pathologischen Eigenschaften als auch die von uns erhobenen molekularen Befunde deuten darauf hin, dass FEA sowohl Indikatorläsionen für ein erhöhtes Risiko hormonabhängiger Mammakarzinome sind als auch ein eigenständiges Progressionsrisiko besitzen und Vorläufer tubulärer Karzinome darstellen.

\section{Korrespondenzadresse \\ Dr. S. Aulmann}

Pathologisches Institut, Universitätsklinikum Heidelberg

Im Neuenheimer Feld 220/221,

69120 Heidelberg

sebastian.aulmann@med.uni-heidelberg.de

Interessenkonflikt. Der korrespondierende Autor gibt an, dass kein Interessenkonflikt besteht.

\section{Literatur}

1. Abdel-Fatah TMA, Powe DG, Hodi Z et al. (2007) High frequency of coexistance of columnar cell lesions, lobular neoplasia and low grade ductal carcinoma in situ with invasive tubular carcinoma and invasive lobular carcinoma. Am J Surg Pathol 31:417-426

2. Aulmann S, Penzel R, Longerich T et al. (2008) Clonality of lobular carcinoma in situ (LCIS) and metachronous invasive breast cancer. Breast Cancer Res Treat 107: 331-335

3. Azzopardi JG (1979) Problems in breast pathology. WB Saunders, Philadelphia, pp 192-213

4. Bratthauer GL, Tavassoli FA (2004) Assessment of lesions coexisting with various grades of ductal intraepithelial neoplasia of the breast. Virchows Arch 444: 340-344

5. Chenna R, Sugawara H, Koike T et al. (2003) Multiple sequence alignment with the Clustal series of programs. Nucleic Acids Res 31: 3497-3500

6. Clamp M, Cuff J, Searle SM, Barton GJ (2004) The Jalview Java Alignment Editor. Bioinformatics 20: 426-427

7. Feeley L, Quinn CM (2008) Columnar cell lesions of the breast. Histopathology 52: 11-19

8. Fernandez-Aguilar S, Simon P, Buxant F et al. (2005) Tubular carcinoma of the breast and associated intra-epithelial lesions: a comparative study with invasive low-grade ductal carcinomas. Virchows Arch 447: 683-687

9. Fraser JL, Raza S, Chorny K et al. (1998) Columnar alteration with prominent apical snouts and secretions: a spectrum of changes frequently present in breast biopsies performed for microcalcifications. Am J Surg Pathol 22: 1521-1527

10. Fritzsche FR, Dietel M, Kristiansen G (2006) Flache epitheliale Atypie und andere Zylinderzell-Läsionen der Brust. Pathologe 27: 381-386

11. Goldstein NS, O'Malley BA (1997) Cancerization of small ectatic ducts of the breast by ductal carcinoma in situ cells with apocrine snouts: a lesions associated with tubular carcinoma. Am J Clin Pathol 107: 561-566

12. Günther K, Merkelbach-Bruse $S$, Amo-Takyi BK et al. (2001) Differences in genetic alterations between primary lobular and ductal breast cancers detected by comparative genomic hybridization. J Pathol 193: 40-47

13. Hwang ES, Nyante SJ, Yi Chen Y et al. (2004) Clonality of lobular carcinoma in situ and synchronous invasive lobular carcinoma. Cancer 100: 25622572

14. Moinfar F, Man YG, Bratthauer GL et al. (2000) Genetic abnormalities in mammary ductal intraepithelial neoplasia-flat type („,clinging ductal carcinoma in situ“). Cancer 88: 2072-2081

15. Morandi L, Marucci G, Foschini MP et al. (2006) Genetic similarities and differences between lobular in situ neoplasia (LN) and invasive lobular carcinoma of the breast. Virchows Arch 449: 14-23

16. O'Malley FP, Mohsin SK, Badve S et al. (2006) Interobserver reproducibility in the diagnosis of flat epithelial atypia of the breast. Mod Pathol 19: 172-179

17. Rosen PP (1999) Columnar cell hyperplasia is associated with lobular carcinoma in situ and tubular carcinoma. Am J Surg Pathol 23: 1561-1562

18. Sahoo S, Recant WM (2005) Triad of columnar cell alteration, lobular carcinoma in situ and tubular carcinoma of the breast. Breast J 11: 140-142
19. Simpson PT, Gale T, Reis-Filho JS et al. (2005) Columnar cell lesions of the breast: the missing link in breast cancer progression? Am J Surg Pathol 29: 734-746 